\title{
Naissance de la critique littéraire, éds. Patrick Dandrey
}

\section{Laura Rescia}

\section{(2) OpenEdition}

1 Journals

\section{Edizione digitale}

URL: http://journals.openedition.org/studifrancesi/4360

DOI: 10.4000/studifrancesi.4360

ISSN: 2421-5856

\section{Editore}

Rosenberg \& Sellier

\section{Edizione cartacea}

Data di pubblicazione: 1 settembre 2016

Paginazione: 325-326

ISSN: 0039-2944

\section{Notizia bibliografica digitale}

Laura Rescia, "Naissance de la critique littéraire, éds. Patrick Dandrey », Studi Francesi [Online], 179 (LXI

II) | 2016, online dal 01 septembre 2016, consultato il 18 septembre 2020. URL : http://

journals.openedition.org/studifrancesi/4360; DOI : https://doi.org/10.4000/studifrancesi.4360

Questo documento è stato generato automaticamente il 18 settembre 2020.

\section{(c) (i) (9)}

Studi Francesi è distribuita con Licenza Creative Commons Attribuzione - Non commerciale - Non opere derivate 4.0 Internazionale. 


\title{
Naissance de la critique littéraire, éds. Patrick Dandrey
}

\author{
Laura Rescia
}

\section{NOTIZIA}

Naissance de la critique littéraire, sous la direction de Patrick DANDREY, «Littératures classiques» 86, 2015, $290 \mathrm{pp}$.

1 L'ipotesi che questo numero di «Littératures classiques» intende dimostrare è che il XVII secolo costituisca il momento cruciale dell'evoluzione del concetto e della pratica di critica letteraria: tra Montaigne e Bayle, tra il lascito dell'Umanesimo, quando essa è ancora e soprattutto glossa e commento erudito, e l'esordio dei Lumi, quando evolverà soprattutto come attività valutativa e analitica, si apre un periodo particolarmente favorevole al suo sviluppo in senso moderno. Perché, come ci ricorda il curatore nell'introduzione al volume, è precisamente in quegli anni che la professionalizzazione dell'attività di scrittore, in parallelo con la specializzazione del sapere, sospinge il suo progresso, articolandola in quattro direzioni; riprendendo Sainte-Beuve, che aveva distinto la critica che "souligne" da quella che "échauffe", il curatore differenzia la pratica assiologica, che giudica e polemizza, da quella programmatica e pragmatica, le cui forme principali sono la poetica e l'ermeneutica. Sono queste le vie che il critico può percorrere quando la sua attività diventa mestiere, e la sua figura quella di arbitro del gusto e del valore, sorta di intermediario "formativo" tra autore e lettore.

2 Questo numero tematico della rivista raccoglie una quindicina di articoli, esito di un ciclo di conferenze tenutesi a Paris-Sorbonne nell'anno accademico 2009-2010, articolate su tre assi di lavoro. Nella prima parte, sono le forme ad essere indagate: vengono studiati $\mathrm{i}$ diversi modi o modelli di discorso che contribuiranno all'elaborazione del genere della scrittura critica. D. FORTIN (Du Parnasse au Panthéon. Les "Vies" d'écrivains du Grand Siècle par leurs contemporains: naissance d'une nouvelle forme critique?, pp. 19-36) ipotizza l'esistenza di un genere ben definito cronologicamente ed 
esteticamente, quello delle vite degli scrittori, situato tra le Vite di ascendenza classica e le biografie, sviluppatesi dopo la Rivoluzione; K. ABIVEN (Fragments d'un discours critique. La question de l'autorité dans les ana, pp. 37-55) si interroga sull'influenza di un genere editoriale, definito dal suffisso -ana, aggiunto al nome dell'autore di cui si collezionano pensieri e aneddoti in queste raccolte, sovente postume, il cui discorso è legittimato dall'evocazione della voce dell'auctoritas, in realtà deformata dall'intervento editoriale; A. ARZOUMANOv (Questionnements éthiques sur le discours critique. L'exemple des clefs d'Ancien Régime, pp. 57-66) si chiede se la pratica delle "chiavi di lettura", che diventa diffusa negli anni 60 del secolo, possa essere considerata una forma di critica, rispondente al desiderio di aprire una nuova strada alla filologia francese o se sia da considerarsi nel novero delle annotazioni ludiche a carattere satirico; B. TEYSSANDIER (Histoire des lettres et tradition de l'erreur. De l'“Avis pour dresser une bibliothèque" de Naudé au "Dictionnaire historique et critique" de Bayle, pp. 67-94) si occupa di un raffronto tra le due opere fondamentali di Naudé e di Bayle, per verificare se l'una possa considerarsi la continuazione dell'altra. Malgrado la condivisione del metodo critico, derivante dal rifiuto dell'errore e della credulità, e la fondamentale dissacrazione dell'auctoritas, l'Avis si inquadrerebbe in una prospettiva storica, una celebrazione del progresso del sapere, mentre il Dictionnaire sarebbe attraversato da una forma più acuta di scetticismo, che rivela l'inquietudine del dubbio e si disvela attraverso l'ironia e la perplessità; L. F. NORMAN (La Querelle des Anciens et des Modernes, ou la métamorphose de la critique, pp. 95-113) affronta la famosa Querelle, esaminando gli strumenti della critica, per rilevare il grado di libertà e autonomia intellettuale che vi si reclama, l'evoluzione del metodo storicista e la crescente complessità della periodizzazione letteraria che ne deriva, nonché il conflitto tra l'eredità razionalista e il nascente approccio sensista.

Nella seconda parte del volume si esaminano le norme, ovvero dei criteri di giudizio elaborati in relazione a un genere specifico o all'interezza dell'attività letteraria: A. DUPRAT (Entre poétique et interprétation. Sur la lettre-préface de Jean Chapelain à l'“Adone" de Marino (1623), pp. 117-128) rimette in discussione la pretesa opposizione tra critica e poetica negli anni Venti e Trenta del secolo, a partire dalla considerazione che spesso le due dimensioni si sovrappongono, e che l'ermeneutica raramente si dissocia dalla teoria estetica: il caso specifico che viene preso in esame evidenzia come la prefazione di Chapelain legittimi l'opera di Marino, restituendola ai lettori francesi, attraverso la sua lettura esegetica, come un'opera conforme alla "regolarità" prima ancora che questa diventasse imperante; C. BARBAFIERI (Du goût, bon et surtout mauvais, pour apprécier l' œuvre littéraire, pp. 129-143), dimostra come, oltre alla regolarità dell'opera letteraria, fin dagli anni Trenta si cominci ad evocare il "mauvais goût", attribuendolo ai pedanti, e fondando il giudizio letterario sul principio del piacere che la comicità scatena. In tal senso, la parodia può essere definita una nuova forma critica, utilizzata per evidenziare i difetti e le mancanze della poesia; C. ESMEIN-SARRAZIN, (Du discours critique à la critique littéraire au XVII siècle: le cas du roman, pp. 145-156), ricordando la condanna del romanzo come genere trasgressivo delle regole della morale e della vraisemblance, sottolinea come la riflessione su questo genere, operata nelle préfaces, nei trattati e nei dibattiti, sposti l'accento dalle accuse precedenti, per interessarsi unicamente alla riuscita o ai difetti intrinseci dell'opera commentata, e introducendo il giudizio personale come criterio di legittimità; M. DUFOUR-MAîTRE (La critique des femmes: le cas des "précieuses", pp. 157-168) rievoca la contestazione molieresca della legittimità dell'autorità femminile in campo critico, squalifica operata tramite la stigmatizzazione del gergo delle preziose. 
Tale interdizione si realizzerebbe anche esaltandone il ruolo idealmente passivo, che sarebbe segnalato da un uso linguistico comune, quale riflesso della Natura; B. PARMENTIER (Le droit à écrire. La Bruyère, "Les caractères" et la critique, pp. 169-184) mette in relazione le affermazioni di La Bruyère sulla critica con la ricezione dei Caractères, dove lo stile è al centro del dibattito, facendo apparire la conflittualità di un ambito letterario in cui è questione di diritti, ovvero di legittimità dell'espressione, laddove la critica letteraria è indissociabile da quella dei costumi e dell'uso linguistico.

Nella terza parte sono presi in esame dei casi specifici, o piuttosto dei singoli autori che si ergono quali sperimentatori delle vie della moderna critica letteraria: M. ROSELLINI (L'entreprise critique de Sorel: une cuvre de "novateur"?, pp. 187-213) esamina l'attività critica di Sorel, che ha attraversato l'intera sua opera: in un primo momento, e notoriamente nei romanzi satirici, la intende come censura, per trasformarla in seguito, nelle sue opere bibliografiche, in analisi della letteratura destinata a formare dei nuovi lettori. Se la preoccupazione estetica è assente dalle sue riflessioni, tutte tese a valutare l'utilità morale e sociale delle belles-lettres, egli anticipa la sociologia del contesto letterario, destinata a un pieno sviluppo nel XIX secolo; B. BEUGNOT ET R. ZUBER, (Guez de Balzac critique pp. 215-229) esaminano la pratica critica di Balzac, articolata in generi diversi, dalla lettera, alla dissertazione all'entretien, il cui stile può essere ricondotto ai termini urbanité e atticisme, uno stile medio che anticipa il registro classico per eccellenza; viene qui analizzata l'epistola XII del suo Epistolarum liber unus, indirizzata a Jean de Silhon, che contiene in particolare la valutazione del ruolo di Malherbe per quanto riguarda la teoria dell'imitazione; B. BEUGNOT (Le Père Bouhours ou de la délicatesse, pp. 231-240) individua la nozione morale ed estetica di delicatesse come fulcro della teoria del gusto e dell'utilizzo del linguaggio del Père Bouhours, nella quale l'armonizzazione dei saperi e della cultura dello scrittore e del lettore presiede all'espressione dell'arte critica; D. REGUIG, (Nicolas Boileau critique, un cas historiographique, pp. 241-258) intende precisare il senso e le modalità critiche di un autore che la vulgata ha troppo spesso appiattito sull'immagine di censore al servizio del classicismo; l'individuazione dei concetti centrali della sua attività critica (principio del gusto, del sublime, e riconoscimento empirico dell'assoluto letterario) consentono di stabilire una continuità tra tale pratica e la sua attività poetica; E. MORTGAT-LONGUET (De l'idéal du critique au discernement du lecteur. Le traité préliminaire aux "Jugements des savants" d'Adrien Baillet, 1685, pp. 259-279) analizza i principi fondativi dell'attività critica secondo Baillet: l'elogio del criterio di valutazione dell'opera letteraria, ispirandosi ai concetti derivanti dall'ideale cartesiano di Port-Royal e di Malebranche, consente la rivalutazione dell'attività di giudizio del critico che, accompagnata da una vasta erudizione e da qualità etiche, conferiscono a questa figura il ruolo di guida per il lettore, messo in guardia contro i critici illegittimi e i pregiudizi. Si inaugura in tal modo l'esercizio della critica dei critici. 